\title{
BMJ Open Resilient healthcare theory as a lens to research emergency department operations: a protocol for a scoping review
}

\author{
Peter Thomas Chessum (D) , ${ }^{1}$ Mark Sujan (D) , ${ }^{2,3}$ Andreas Xyrichis (D) , \\ Janet E Anderson (D) ${ }^{4}$
}

To cite: Chessum PT, Sujan M, Xyrichis $\mathrm{A}$, et al. Resilient healthcare theory as a lens to research emergency department operations: a protocol for a scoping review. BMJ Open 2021;11:e053701. doi:10.1136/ bmjopen-2021-053701

- Prepublication history for this paper is available online To view these files, please visit the journal online (http://dx.doi. org/10.1136/bmjopen-2021053701).

Received 20 May 2021 Accepted 08 December 2021

Check for updates

(c) Author(s) (or their employer(s)) 2021. Re-use permitted under CC BY-NC. No commercial re-use. See rights and permissions. Published by BMJ.

${ }^{1}$ Florence Nightingale Faculty of Nursing, Midwifery \& Palliative Care, King's College London, London, UK

${ }^{2}$ Warwick Medical SchoolSocial Sciences and Systems in Health, University of Warwick,

Coventry, UK

${ }^{3}$ Human Factors Everywhere

LTD, Woking, UK

${ }^{4}$ School of Health Sciences, City University, London, UK

Correspondence to

Mr Peter Thomas Chessum;

Peter.chessum@kcl.ac.uk

\section{ABSTRACT}

Introduction Emergency departments (EDs) are complex systems that have constant fluctuations in demand, creating mismatches with planned capacity. Despite the complexity of ED operations, quality and safety improvement are often approached in a reactive, linear and reductionist manner. There is increasing interest in adopting Resilient Healthcare (RHC) techniques based on complex systems thinking as a method for quality improvement and research in EDs. However, the evidence for this approach is still developing and it is not clear what techniques have been used so far and which are most effective. This scoping review will be conducted between March 2022 until May 2022. It seeks to examine the international literature for available reports that have adopted RHC theory to study ED operations and identify approaches used and proposed benefits.

Methods and analysis The methodology for scoping reviews outlined by Arksey and 0'Malley (2005) will be followed, acknowledging refinements made to the scoping review process by Levac et al (2010). The methodology consists of five steps: (1) identifying the research question; (2) identifying the relevant literature; (3) study selection; (4) charting the data; and (5) collating, summarising and reporting the results. A two-stage approach will be undertaken to synthesise and report results: (1) numerical analysis of the nature and distribution of studies (the overall number of studies, country of origin, the most studied core function of ED, type of research design); and (2) a thematic mapping of the literature.

Ethics and dissemination Scoping review methodology synthesises published data and, therefore, does not require ethical approval. An article formatted in line with Preferred Reporting Items for Systematic Reviews and Meta-Analyses forScoping Reviews reporting guidance will be submitted for publication to a scientific journal. Findings will also be presented at relevant advanced practice conferences and disseminated within clinical and academic groups.

\section{INTRODUCTION}

Emergency departments (EDs) can be described as 'socio-technical' systems. ${ }^{1-3}$ Work is achieved using highly specialised medical technology, information systems and
Strengths and limitations of this study

- Uses a systematic strategy to identify and map out literature to inform understanding of resilient healthcare approaches to research and quality improvement in emergency departments.

- Scoping study methodology allows for the inclusion of a wide range of study designs.

- Scoping studies do not typically assess the quality of the evidence identified in the literature.

- Grey literature will not be included in this review.

- The research team is multidisciplinary with expertise in emergency care, advanced practice, leadership, systematic reviewing and resilient healthcare.

the social co-ordination of multiple professionals from different disciplines with highly technical knowledge and skills. ${ }^{1-3}$ EDs are also complex adaptive systems, with permeable operational boundaries that mean they are affected by fluctuations in demand and capacity, requiring constant adaptations and adjustments to processes to ensure consistent levels of care. ${ }^{2}{ }^{3}$ Despite the complexity of operations, research and quality improvements are often only approached in a reactive, linear and reductionist manner. ${ }^{3} 4$ As an example, crowding has frequently been described in terms of 'input-throughputoutput' but simplifying this phenomenon's complexity may impede our understanding and reduce our ability to make safety improvements. ${ }^{1-3}$ There is a new approach to researching, learning and improving safety and quality in complex systems termed Resilient Healthcare (RHC) theory, which is based on understanding this complexity and designing interventions to support adaptation. $^{1-8}$

\section{Background}

Resilience has been a topic of interest in various disciplines, including health, 
sociology, psychology, engineering, economics and political science. ${ }^{9}$ In healthcare, resilience has previously been used to describe individual psychological resilience to stress and pressure ${ }^{10}$ RHC differs from this; it is used to characterise a system's ability to adjust performance to meet demands by responding to changes, opportunities or disturbances in conditions in a flexible and timely manner. ${ }^{10}{ }^{11}$ The purpose of exploring the resilience of a system is to increase the number of acceptable outcomes which in turn is theorised to reduce unacceptable outcomes. ${ }^{12}{ }^{13}$ System resilience is proposed to be underpinned by four resilience potentials or activities ${ }^{14}$ :

1. Responding. Knowing what to do or being able to respond to regular or irregular changes, disturbances or opportunities by adjusting functioning or by creating new ways of doing things.

2. Monitoring. Knowing what to look for or being able to monitor that which affects or could affect an organisation's performance in the near term-positively or negatively.

3. Learning. Knowing what has happened or being able to learn from experience, in particular, to learn the right lessons from the right experiences.

4. Anticipating. Knowing what to expect or being able to anticipate developments further into the future, such as potential disruptions.

(Hollnagel, 2009)

Anderson and Ross have proposed a fifth resilience activity, coordinating, which highlights the importance of team members working together to communicate and deliver care. ${ }^{15}$ RHC proposes that strengthening these activities can strengthen a system's performance under expected or unexpected conditions. ${ }^{15}$ Resilience cannot be directly observed during everyday operations and is only apparent in hindsight when analysing difficult situations and how they were managed. ${ }^{12}$ The resilience potentials, or activities can be reviewed at any time to improve safety and quality proactively. ${ }^{13}$ This implies that healthcare organisations could switch from traditional investigative processes, only learning from rare adverse outcomes to an approach examining how, and why under varying conditions, healthcare generally succeeds. ${ }^{12} 131516$

EDs rely on workers to adapt to challenges, work around problems and devise innovative solutions to maintain safety. ${ }^{1-6}$ Historically, safety improvement efforts have been based on constraining adaptations through standardised procedures and policies, with little consideration of the contextual factors that demand flexible worker responses. ${ }^{2}{ }^{15}$ Individuals may learn successful adaptations from observing other staff in the ED, but systematic organisational learning on adaptive capacity is routinely lacking. ${ }^{3}$ It is important to understand local individual or team adaptations that result in good outcomes as it may highlight opportunities for further system improvement. ${ }^{3}$ Reliance on staff to maintain routine operations despite fluctuating and variable conditions could be suboptimal if system design is not also addressed. ${ }^{1718}$ The constant expectation of staff to make adaptations in clinical situations deviating from normal working practices to meet demands can result in frustration, cynicism, burnout and high turnover in staff. ${ }^{18}$ Workers' adaptations can also hide issues from managers, which impairs their ability to make system changes ${ }^{17}$. Implementing RHC in EDs could mitigate these effects and strengthen the emergency care system, but the practical steps involved, how they have been implemented, and the proposed benefits remain unclear.

This scoping review will be beneficial to both emergency medicine and resilient healthcare communities. RHC is an area of growing interest, but there remains an increasing desire to operationalise its concepts and demonstrate its workable interventions in healthcare settings. ${ }^{19}$ Emergency care has been a focus for RHC studies exhibiting promise in how to understand clinical practices in complex adaptive systems. ${ }^{3}$ There are now sufficient studies to warrant a scoping review of the contribution of RHC to improving emergency care. Consolidation of RHC theory used in ED may improve clinicians' and managers' understanding of safety and quality improvement from a broader system perspective. A new account of which aspects of emergency care have or have not been explored and whether RHC has contributed new insights and improvements to ED will identify gaps in the current literature base. This is essential due to emergency care's high hazard and dynamic nature and the need to identify new recommendations enabling safety and service improvements.

\section{METHOD}

The methodology for scoping studies outlined by Arksey and O'malley will be followed, acknowledging refinements made to the scoping review process by Levac et $a l^{20}{ }^{21}$ The methodology consists of five steps: (1) identifying the research question; (2) identifying the relevant literature; (3) study selection; (4) charting the data; and (5) collating, summarising and reporting the results.

\section{Stage 1: developing the research question}

This scoping review has been designed to provide a broad overview of how RHC researchers have studied emergency care, implemented RHC and the proposed benefits. Scoping reviews are used to identify gaps in evidence and clarify key concepts in research where it is not desirable to identify a narrow research question. ${ }^{20}$ There are four research questions:

1. What aspects of ED operations have been studied using RHC?

2. What study designs and methods have been used to study RHC in EDs?

3. What are the proposed benefits of using an RHC perspective to study EDs?

4. What interventions have been proposed following RHC studies of EDs?

\section{Stage 2: identifying relevant studies}

Initial scoping of the literature showed that empirical studies of RHC interventions were rare and exploratory 
studies, including case studies and practice reports, were common. Therefore, we will seek empirical evidence or academic contributions that have used RHC in EDs, including primary research, systematic reviews and book chapters. Many academic RHC contributions are published within a series of RHC textbooks that have been identified by experts. These books will be sourced, chapter titles relevant to the scoping review identified and placed within an Excel spreadsheet to categorise and avoid duplication before data extraction.

PTC will develop the database search strategy using an iterative three-step approach. Search terms are (combining population, context and concept through Boolean operators AND, and OR within each category respectively): ["Emergency room" OR "ER" OR "Accident and Emergency" OR "A\&E” OR "Emergency Department" OR "ED"] AND ["Resilient Healthcare" OR "Resilient Health Care" OR "Resilience engineering" OR "Safety*I" OR "Safety*1" OR "Safety*2" OR "Safety*II" OR "Workas-imagined" OR "Work- as- done"]. PTC piloted these search terms on one database the Cumulative Index to Nursing and Allied Health Literature (CINAHL). Initial articles will be reviewed, and keywords refined if required; the search will then be rerun accordingly. Once the search terms are finalised, full searches on other relevant databases (CINAHL, Medline, Embase, Web of Science, Scopus) will be performed. Covidence bibliographic software will be used to manage records. ${ }^{22}$

\section{Stage 3: study selection}

A systematic approach to selecting studies will be used and reported using the Preferred Reporting Items for Systematic Reviews and Meta-Analyses (PRISMA)-ScR flow chart. ${ }^{23}$ Before data extraction, the research team will adopt a three-stage process using Covidence software to review articles against eligibility criteria (table 1).

Databases will be searched and all titles will be uploaded to the Covidence platform. An initial title screening of all papers using the 'include', 'maybe' or 'exclude' function in the Covidence programme will be performed (PTC). Ten per cent of the rejected titles will be screened by RHC experts JA (5\%) and MS (5\%) to check for agreement, with the possibility to reinstate titles previously excluded at the title screen. At stage three after title screening, all full text papers will be uploaded to the Covidence software for team review. PTC, JA and MS will review all of the full texts; with two votes required on full texts to progress to final data extraction. It is expected that the search will highlight many papers that relate to resilience from a psychological perspective; these will be excluded, as they do not relate to resilient healthcare or resilience engineering. The majority of works on resilient healthcare are published within English speaking countries, but any published in languages other than English will also be excluded.

\section{Stage 4: data collection}

A draft data extraction form has been developed, which includes the following items: (1) authors, (2) year of publication, (3) country of origin, (4) aims/objectives, (5) ED operation under review, (6) identifiable RHC concepts, (7) method used for data collection, (8) key findings and (9) suggested interventions. To identify resilient healthcare concepts applied in EDs, the resilience potentials of anticipating, monitoring, responding, coordinating and learning that are defined by authors in the text will be extracted. Additional data extraction categories that have been identified in previous research will also be used if applicable. These are 'misalignments', 'variability', 'adaptations' and 'goal trade-offs'. ${ }^{4}$ The data extraction form will be trialled on a small number of papers $(n=5)$ revised if necessary and then used for charting all papers and chapters. Scoping review methodology states that data extraction is an iterative process, and in keeping with this charting will be revised further if required during any stage of the process. ${ }^{20}{ }^{21}$ Appraisal of individual studies' quality is not commonly performed within scoping reviews ${ }^{20} 21$ and will not be used in this review. However, study designs used and type of evidence will be collated.

\section{Stage 5: data summary and synthesis of results}

A two-stage approach will be undertaken to analyse and report results: (1) numerical analysis of the nature and

Table 1 Inclusion and exclusion criteria

\begin{tabular}{|c|c|}
\hline Inclusion criteria & Exclusion criteria \\
\hline $\begin{array}{l}\text { Types of publication: original research that reports empirical } \\
\text { studies or academic outputs (eg, studying resilience, } \\
\text { or applying or discussing resilience theory) on RHC in } \\
\text { emergency departments. } \\
\text { Context: hospital emergency departments } \\
\text { Concept/Core focus: applying resilient healthcare theory. } \\
\text { English language. }\end{array}$ & $\begin{array}{l}\text { Types of publication: dissertations/theses; reports or } \\
\text { abstracts only; letters to editors; conference proceedings; } \\
\text { reviews or editorials. } \\
\text { Individual or team psychological resilience. } \\
\text { Community resilience (to respond to or withstand } \\
\text { adverse situations, for example, energy, communication, } \\
\text { transportation, collapse) } \\
\text { Information technology resilience. } \\
\text { Disaster recovery. } \\
\text { Studies where 'resilience' is only mentioned briefly or } \\
\text { descriptively. }\end{array}$ \\
\hline
\end{tabular}

RHC, Resilient Healthcare. 
distribution of studies (overall number of studies, country of origin, most studied core function of ED, type of research design); and (2) the research team will map out clusters of papers on aspects of emergency care reviewed using an RHC lens. Narrative synthesis will then be used to identify, compare and contrast common themes in the data.

\section{Dissemination and ethics}

As this review will only include published data, ethics approval will not be sought. This study will examine and map out the literature on RHC concepts applied in EDs. An article formatted in line with PRISMA-ScR reporting guidance will be submitted for publication to a scientific journal. Findings will also be presented at relevant advanced practice conferences and disseminated within clinical and academic groups.

Twitter Peter Thomas Chessum @LEADACP, Mark Sujan @MarkSujan, Andreas Xyrichis @AndreasXyrichis and Janet E Anderson @ProfAndersonHF

Contributors PTC contributed to the development of the research question and research design and drafted the initial and subsequent versions of the manuscript. $\mathrm{JA}, \mathrm{MS}$ and $\mathrm{AX}$ contributed to the development of the research question, research design and provided input to draft versions of the manuscript.

Funding The authors have not declared a specific grant for this research from any funding agency in the public, commercial or not-for-profit sectors.

Competing interests None declared.

Patient and public involvement Patients and/or the public were not involved in the design, or conduct, or reporting, or dissemination plans of this research.

Patient consent for publication Not applicable.

Provenance and peer review Not commissioned; externally peer reviewed.

Open access This is an open access article distributed in accordance with the Creative Commons Attribution Non Commercial (CC BY-NC 4.0) license, which permits others to distribute, remix, adapt, build upon this work non-commercially, and license their derivative works on different terms, provided the original work is properly cited, appropriate credit is given, any changes made indicated, and the use is non-commercial. See: http://creativecommons.org/licenses/by-nc/4.0/.

\section{ORCID iDs}

Peter Thomas Chessum http://orcid.org/0000-0002-2701-1901

Mark Sujan http://orcid.org/0000-0001-6895-946X

Andreas Xyrichis http://orcid.org/0000-0002-2359-4337

Janet E Anderson http://orcid.org/0000-0002-1452-8370

\section{REFERENCES}

1 Nugus P, Forero R. Understanding interdepartmental and organizational work in the emergency department: an ethnographic approach. Int Emerg Nurs 2011;19:69-74.

2 Braithwaite J, Clay-williams R, Nugus P. Health care as a complex adaptive system. In: Hollnagel E, Braithwaite J, Wears R, eds.
Resilient healthcare. Boca Raton: CRC Press: Taylor Francis Group, 2013: 57-73.

3 Braithwaite J, Clay-Williams R, Hunte G. Understanding resilient clincial practices in emergency department ecosystems. In: Braithwaite J, Wears R, Hollnagel E, eds. Resilient health care: reconciling Work-as-Imagined and Work-as-Done. New York: CRC publishing, 2019: 2019. 89-102.

4 Anderson JE, Ross AJ, Back J, et al. Beyond 'find and fix': improving quality and safety through resilient healthcare systems. Int J Qual Health Care 2020;32:204-11.

5 Back J, Ross AJ, Duncan MD, et al. Emergency department escalation in theory and practice: a mixed-methods study using a model of organizational resilience. Ann Emerg Med 2017;70:659-71.

6 Nugus P, Forero R, McCarthy S, et al. The emergency department "carousel": an ethnographically-derived model of the dynamics of patient flow. Int Emerg Nurs 2014;22:3-9.

7 Hunte G, Marsden J. Engineering resilience in an urban emergency department. In: Hollangel E, Braithwaite J, Wears R, eds. Delivering resilient health care. Oxon: Routledge, 2019: 132-49.

8 Hunte G. A lesson in resilience: the 2011 stanley cup riot. In: Wears R, Hollnagel E, Braithewaite J, eds. Resilient health care: the resilience of everyday clinical work. Surrey: Ashgate, 2015: vol 2. 1-9.

9 Wiig S, Fahlbruch B. Exploring resilience: a scientific journey from practice to theory. 1 edn. Berlin: Springer Nature Switzerland AG, 2019.

10 Anderson J, Ross A, Back J. Resilience Engineering for quality improvement. In: Hollnagel E, Braithwaite J, Wears R, eds. Delivering resilient healthcare. Abingdon: Routledge, 2019: 32-43.

11 Anderson JE, Ross AJ, Back J, et al. Implementing resilience engineering for healthcare quality improvement using the care model: a feasibility study protocol. Pilot Feasibility Stud 2016;2.

12 Hollnagel E. Safety-I and Safety-II: the past and future of safety management. 1 edn. CRC Press Surrey, 2014.

13 Hollnagel E. Safety-II in practice: developing the resilience potentials. 1 edn. Oxon: Routledge, 2018.

14 Hollnagel E. The four cornerstones of resilience engineering. In: Resilience engineering perspectives Prepartion and restoration. Farnham: Ashgate, 2009: vol 2. 117-34.

15 Anderson JE, Ross AJ. A Handbook for improving quality through resilient systems, 2020Centre for Applied Resilience in Healthcare. Available: https://resiliencecentre.org.uk/care-qi-handbook/ [Accessed 18 May 2021].

16 Sujan M, Pozzi S, Valbonesi V. Reporting and learning: from extraordinary to ordinary. In: Braithwaite J, Wears R, Hollnagel E, eds. Resilient health care: reconciling Work-as-Imagined and Workas-Done. New York: CRC publishing, 2017: 2019. 103-10.

17 Debono D, Clay-Williams R, Taylor N, et al. Using workarounds to examine characteristics of resilience in action. In: Hollnagel $\mathrm{E}$, Braithwaite J, Wears R, eds. Delivering resilient health care. Oxon: Routledge, 2019: 44-55.

18 Wears R, Vincent CCare RH, ed. Relying on resilience: too much of a good thing? Boca Raton: CRC Press, 2013: 135-44.

19 Iflaifel M, Lim RH, Ryan K, et al. Resilient health care: a systematic review of conceptualisations, study methods and factors that develop resilience. BMC Health Serv Res 2020;20:324.

20 Arksey H, O'Malley L. Scoping studies: towards a methodological framework. Int J Soc Res Methodol 2005;8:19-32.

21 Levac D, Colquhoun H, O'Brien KK. Scoping studies: advancing the methodology. Implement Sci 2010;5:69.

22 Covidence. Main steps in Covidence, 2019. Available: https:// support.covidence.org/help/getting-started-with-covidence

23 Tricco AC, Lillie E, Zarin W, O'Brien W, et al. PRISMA extension for scoping reviews (PRISMA-ScR): checklist and explanation. Ann Intern Med 2018;169:467-73.

24 Pham MT, Rajić A, Greig JD, et al. A scoping review of scoping reviews: advancing the approach and enhancing the consistency. Res Synth Methods 2014;5:371-85. 